\title{
Controlling cholesterol entry into mitochondria, a key step for hepatocarcinogenesis in non-alcoholic steatohepatitis-related hepatocellular carcinoma
}

\author{
Guillermo Daniel Mazzolini $^{1,2} \wedge$, Eda Kaya $^{3,4} \wedge$, Ali Canbay $^{3,4} \wedge$ \\ ${ }^{1}$ Gene Therapy Laboratory, Instituto de Investigaciones en Medicina Traslacional (IIMT), Universidad Austral-CONICET, Buenos Aires, Argentina; \\ ${ }^{2}$ Liver Unit, Hospital Universitario Austral, Universidad Austral, Buenos Aires, Argentina; ${ }^{3}$ Section of Gastroenterology and Hepatology, University \\ Hospital Knappschaftskrankenhaus Bochum, Ruhr University Bochum, Bochum, Germany; ${ }^{4}$ Department of Medicine, University Hospital \\ Knappschaftskrankenhaus Bochum, Ruhr University Bochum, Bochum, Germany \\ Correspondence to: Ali Canbay, MD. Professor of Medicine, Director of the Department of Medicine, Ruhr-University Bochum, University Hospital \\ Knappschaftskrankenhaus Bochum, In der Schornau 23-25, 44892 Bochum, Germany. Email: ali.canbay@rub.de. \\ Comment on: Conde de la Rosa L, Garcia-Ruiz C, Vallejo C, et al. STARD1 promotes NASH-driven HCC by sustaining the generation of bile acids \\ through the alternative mitochondrial pathway. J Hepatol 2021;74:1429-41.
}

Submitted Sep 28, 2021. Accepted for publication Oct 22, 2021.

doi: $10.21037 / \mathrm{hbsn}-21-406$

View this article at: https://dx.doi.org/10.21037/hbsn-21-406

Hepatocellular carcinoma (HCC) is the sixth most common cancer and the second leading cause of cancer-related death worldwide (1). Unfortunately, the incidence and mortality of HCC are increasing steadily in formerly lowrisk countries (2). Although the contribution of patients infected with hepatitis $\mathrm{B}$ virus (HBV) and hepatitis $\mathrm{C}$ virus (HCV) has dramatically decreased due to the use of highly effective antiviral agents, increasing epidemiologic evidence indicates a link between non-alcoholic fatty liver disease (NAFLD) and the risk of HCC (1). It is important to highlight that the prevalence of NAFLD has increased in parallel with the obesity, metabolic syndrome, and type 2 diabetes mellitus pandemic. Among patients with NAFLD, the incidence of HCC is around 0.44 per 1,000 person-years (3). The identification of the pathogenic mechanisms linking NAFLD and especially non-alcoholic steatohepatitis (NASH) to HCC is still a matter of study. Of particular importance in NASH-driven HCC is that a substantial proportion of patients have minimal or even no signs of liver fibrosis (4). In comparison with $\mathrm{HCV}$, the risk of developing $\mathrm{HCC}$ is increased 5 -fold in NAFLD and metabolic syndrome in non-cirrhotic patients (5).
Despite the advances in therapeutic strategies (immunostimulatory monoclonal antibodies, the PD-1 inhibitors atezolizumab and bevacizumab), the 5-years overall survival of patients with HCC in all stages is still poor, about $18 \%$ (1). Therefore, it is clear that new effective therapies are urgently needed for this devastating disease. Deeper insight into pathophysiology and mechanisms involved in hepatocarcinogenesis will definitively contribute to cover this gap. Still, the mechanisms of HCC development in NAFLD/NASH remain unclear and deserve profound investigation at the molecular level.

From the physiological point of view, synthesis of bile acids (BAs) in the liver is the major pathway for cholesterol output, and BAs are secreted into the bile to facilitate fat and vitamin digestion, but BAs also regulate metabolism, inflammatory responses, and gut microbiota. In addition, the association between HCC development and BAs metabolism has been demonstrated experimentally and in clinical settings (6-8).

BAs are synthesized from cholesterol in the liver through two main pathways, the classical/neutral and the alternative/acidic pathway. In the classical pathway, BAs are synthesized from cholesterol involving cholesterol-7-alpha-

^ ORCID: Guillermo Daniel Mazzolini, 0000-0002-1450-8441; Eda Kaya, 0000-0002-9293-2811; Ali Canbay, 0000-0001-6069-7899. 
hydroxylase (CYP7A1), the initial and rate-determining step in this pathway of BA synthesis. Another important step is the conversion of $7 \alpha$-hydroxy-4-cholestene-3one by sterol $12 \alpha$-hydroxylase (CYP8B1) to cholic acid (CA). In the alternative pathway, cholesterol is oxidized by mitochondrial sterol 27-hydroxylase (CYP27A1), located in the inner mitochondrial membrane (which contains very low cholesterol amounts), and its products are further hydroxylated by oxysterol $7 \alpha$-hydroxylase (CYP7B1) with a subsequent enzymatic conversion to generate chenodesoxicolic acid (CDCA). The mitochondrial cholesterol level and therefore BAs metabolism is regulated by steroidogenic acute regulatory protein 1 (STARD1), but the alternative pathway of BAs synthesis has a minor contribution to the BAs pool (9).

The BAs homeostasis is regulated by the farnesoid $\mathrm{X}$ receptor (FXR) and G-protein coupled BA receptor 1 (GPBAR1/TGR5) (10). FXR plays a key role in regulating lipid metabolism and inflammation in the liver, and studies have shown that FXR expression is reduced in human HCC (11). Although we and others have shown that serum BA concentrations are elevated in advanced NASH, and that BAs accumulation induces liver damage and contributes to the progression of NAFLD, the role of the alternative BAs pathway in HCC development was not previously explored.

The work from Conde de la Rosa et al. elegantly demonstrated a key role for STARD1 in NASH-related HCC by the stimulation of mitochondrial BAs synthesis which further induced self-renewal and stemness in tumor-initiated stem-like cells (12). They first showed in patients with NASH-driven HCC an increased expression of STARD1 and BAs in liver tissue in comparison with controls. Then, they established a complex animal model, consisting of DEN-pretreated mice with a high-fat diet (HFD) supplemented with cholesterol to assess the effects on STARD1 expression and hepatocarcinogenesis; a more severe fibrosis degree was induced when compared to mice not supplemented with cholesterol, and the addition of cholesterol promoted NASH-related HCC and induced STARD1 expression. In addition, treatment with ezetimibe, an inhibitor of cholesterol absorption which previously showed anti-HCC effects (13), reduced tumor burden and increased animal survival, highlighting the role of cholesterol in the promotion of HCC development in the model studied.

In patients with NASH the presence of high levels of cholesterol and over-expression of STARD1 was previously reported by the authors (14), although, the role of STARD1 in hepatocarcinogenesis was not explored until now. The key role of STARD1 in HCC development was elegantly studied with knock-in and knock-down experiments in mice. Moreover, the effects of NASH-derived BAs profiles were investigated in tumor-initiated stem-like cells and primary mouse hepatocytes, where Conde de la Rosa et al. demonstrated that BAs stimulate the expression of transcription factors involved in self-renewal and pluripotency, which are key for HCC development (12).

Conde de la Rosa et al. provide a new mechanism of hepatocarcinogenesis in mice which involves the activation of the alternative BAs synthesis pathway (12). The authors could show a correlation between total BAs and the expression of STARD1 in humans and one might speculate that the activation of the alternative BAs synthesis pathway in metabolic syndrome, type 2 diabetes, obesity and NAFLD/ NASH might be involved in the pathophysiology of HCC development. Therefore, is there any role for anticholesterol therapy for the chemoprevention of HCC development in NAFLD/NASH patients? In this regard, a recent systematic review and meta-analysis showed that use of statins was safe and associated with a lower pooled risk of HCC development among adults with chronic liver diseases (15).

In summary, controlling cholesterol entry into mitochondria might possibly inhibit a key step for hepatocarcinogenesis in NASH-related HCC.

\section{Acknowledgments}

Funding: This project was funded by the Wilhelm Laupitz Foundation and the German Research Foundation (DFG CA267/13-3) to AC.

\section{Footnote}

Provenance and Peer Review: This article was commissioned by the editorial office, Hepatobiliary Surgery and Nutrition. The article did not undergo external peer review.

Conflicts of Interest: All authors have completed the ICMJE uniform disclosure form (available at https://hbsn. amegroups.com/article/view/10.21037/hbsn-21-406/coif). The authors have no conflicts of interest to declare.

Etbical Statement: The authors are accountable for all aspects of the work in ensuring that questions related 
to the accuracy or integrity of any part of the work are appropriately investigated and resolved.

Open Access Statement: This is an Open Access article distributed in accordance with the Creative Commons Attribution-NonCommercial-NoDerivs 4.0 International License (CC BY-NC-ND 4.0), which permits the noncommercial replication and distribution of the article with the strict proviso that no changes or edits are made and the original work is properly cited (including links to both the formal publication through the relevant DOI and the license). See: https://creativecommons.org/licenses/by-nc-nd/4.0/.

\section{References}

1. Llovet JM, Kelley RK, Villanueva A, et al. Hepatocellular carcinoma. Nat Rev Dis Primers 2021;7:6.

2. Sung H, Ferlay J, Siegel RL, et al. Global Cancer Statistics 2020: GLOBOCAN Estimates of Incidence and Mortality Worldwide for 36 Cancers in 185 Countries. CA Cancer J Clin 2021;71:209-49.

3. Younossi ZM, Koenig AB, Abdelatif D, et al. Global epidemiology of nonalcoholic fatty liver disease-Metaanalytic assessment of prevalence, incidence, and outcomes. Hepatology 2016;64:73-84.

4. Ertle J, Dechêne A, Sowa JP, et al. Non-alcoholic fatty liver disease progresses to hepatocellular carcinoma in the absence of apparent cirrhosis. Int J Cancer 2011;128:2436-43.

5. Mittal S, El-Serag HB, Sada YH, et al. Hepatocellular Carcinoma in the Absence of Cirrhosis in United States Veterans is Associated With Nonalcoholic Fatty Liver Disease. Clin Gastroenterol Hepatol 2016;14:124-31.e1.

6. Wang X, Fu X, Van Ness C, et al. Bile Acid Receptors and Liver Cancer. Curr Pathobiol Rep 2013;1:29-35.

7. Sydor S, Best J, Messerschmidt I, et al. Altered Microbiota
Diversity and Bile Acid Signaling in Cirrhotic and Noncirrhotic NASH-HCC. Clin Transl Gastroenterol 2020;11:e00131.

8. Bechmann LP, Kocabayoglu P, Sowa JP, et al. Free fatty acids repress small heterodimer partner (SHP) activation and adiponectin counteracts bile acid-induced liver injury in superobese patients with nonalcoholic steatohepatitis. Hepatology 2013;57:1394-406.

9. Miller WL. Steroidogenic acute regulatory protein (StAR), a novel mitochondrial cholesterol transporter. Biochim Biophys Acta 2007;1771:663-76.

10. Arab JP, Karpen SJ, Dawson PA, et al. Bile acids and nonalcoholic fatty liver disease: Molecular insights and therapeutic perspectives. Hepatology 2017;65:350-62.

11. Su H, Ma C, Liu J, et al. Downregulation of nuclear receptor FXR is associated with multiple malignant clinicopathological characteristics in human hepatocellular carcinoma. Am J Physiol Gastrointest Liver Physiol 2012;303:G1245-53.

12. Conde de la Rosa L, Garcia-Ruiz C, Vallejo C, et al. STARD1 promotes NASH-driven HCC by sustaining the generation of bile acids through the alternative mitochondrial pathway. J Hepatol 2021;74:1429-41.

13. Miura K, Ohnishi H, Morimoto N, et al. Ezetimibe suppresses development of liver tumors by inhibiting angiogenesis in mice fed a high-fat diet. Cancer Sci 2019;110:771-83.

14. Caballero F, Fernández A, De Lacy AM, et al. Enhanced free cholesterol, SREBP-2 and StAR expression in human NASH. J Hepatol 2009;50:789-96.

15. Wong YJ, Qiu TY, Ng GK, et al. Efficacy and Safety of Statin for Hepatocellular Carcinoma Prevention Among Chronic Liver Disease Patients: A Systematic Review and Meta-analysis. J Clin Gastroenterol 2021;55:615-23.
Cite this article as: Mazzolini GD, Kaya E, Canbay A. Controlling cholesterol entry into mitochondria, a key step for hepatocarcinogenesis in non-alcoholic steatohepatitisrelated hepatocellular carcinoma. HepatoBiliary Surg Nutr 2021;10(6):890-892. doi: 10.21037/hbsn-21-406 